\title{
Dualities or Analogies between Superstrings and Multi-fold Universes
}

\author{
Stephane H. Maes ${ }^{1}$
}

June 14, 2020

\begin{abstract}
:
Superstrings seem to somehow appear in multi-folds universes. We compare the results and models of superstrings with the multi-fold mechanisms associated to EPR entanglement and discover that such multi-fold mechanism in a multi-fold universe: i) explain or clarify many superstring results, ii) provide analogies to superstring results that often change conjectures (e.g. AdS/CFT correspondence, ER=EPR, GR=QM) to facts or theorems in a multi-fold universe iii) position superstrings with respect to our spacetime iv) illustrates differences and $v$ ) makes suggestions on how to evolve superstrings theories and M-Theory, according to the multi-fold universe proposal and observations in the real universe, so far. We conclude with a call for collaboration.
\end{abstract}

\section{Introduction}

The new preprint [1] proposes contributions to several open problems in physics like the reconciliation of General Relativity with Quantum Physics, explaining the origin of gravity proposed as emerging from quantum (EPREinstein Podolsky Rosen) entanglement between particles, detailing contributions to dark matter and dark energy and explaining other Standard Model mysteries without requiring New Physics beyond the Standard Model other than the addition of gravity to the Standard Model Lagrangian. All this is achieved in s multi-fold universe that may well model our real universe, which remains to be validated.

With the proposed model of [1], spacetime and Physics are modeled from Planck scales to quantum and macroscopic scales and semi classical approaches appear valid till very small scales. In [1], it is argued that spacetime is discrete, with a random walk-based fractal structure, fractional and noncommutative at, and above Planck scales (with a 2-D behavior and Lorentz invariance preserved by random walks till the early moments of the universe). Spacetime results from past random walks of particles. Spacetime locations and particles can be modeled as microscopic blackholes (Schwarzschild for photons and spacetime coordinates, and metrics between Reisner Nordstrom [24] and Kerr Newman [25] for massive and possibly charged particles - the latter being possibly extremal). Although surprising, [1] recovers results consistent with other like [32], while also being able to justify the initial assumptions of black holes from the gravity or entanglement model. The resulting gravity model recovers General Relativity at larger scale, as a 4-D process, with massless gravity, but also with massive gravity components at very small scale that make gravity significant these scales. Semi-classical models also work well till way smaller scales that usually expected.

Throughout the analysis, [1] finds numerous touch points with superstrings, despite coming from a complete different proposition, i.e. 1) not starting from General Relativity (GR) or the Hilbert Einstein Action adapted for higher dimensions (or variations and extensions) 2) no modeling strings and not starting from a string equation or a string action like the Nambu-Goto Action 3) not declaring any supersymmetry or supergravity invariance 4) in fact, delaying quantization as long as possible in the discussions of the multi-fold mechanisms.

${ }^{1}$ shmaes.physics@gmail.com 
In this paper, we remain at a high level of discussion of the analysis. It makes the points accessible to a wider audience and keeps the door open to further papers or discussions devoted to details of interest. Yet it requires the reader to review [1], as we do not revisit here all the details of the multi-fold mechanism or reconstruction of spacetime. The followings subsections are organized as a series of observations in [1] that relate to superstrings and conversely. [1] did not focus on presenting these facts as a comparison or as lessons learned for and from superstrings. Also, our analysis is not exhaustive. However, we hope that it will intrigue enough the reader to push him or her to dig deeper. Most of the more detailed (or entry point) references are provided in [1] and so every statement is not motivated here or presented with the most appropriate references. This paper is rather a story tale. "[1]" appears often, as a person or a model, to refer to the original arguments, analysis, mechanisms or proposals discussed in [1].

[1] is about a multi-fold universe. We discovered that the framework and mechanism of [1] have many touch points with superstrings, in terms of the resulting picture of the universe and specific properties or phenomena. [1] argues that it can model well our real space time and provides predictions and opportunities for falsifiability.

\section{Selected duality highlights}

\subsection{Point particles, strings, world sheets and black holes}

[1] works with a model where, from the beginning, particles are central to the analysis (at the difference of conventional QFT, which has many modeling problems with particles). The suitability of the approach comes from the strict rejection of any supra luminosity in a multi-fold universe defined by [1]. Accordingly, for example, Path Integrals must filter out any path that would have a portion space like with respect to another portion of the path. This is a key difference with conventional QFT. It avoids excessive spread of the wave functions of relativistic particles, and zeroes QFT correlations between space like regions. Of course, it is understood that particles are only tractable between their creation and annihilation and that their numbers change.

In addition, from the onset, a particle in [1] is associated to an uncertainty region, according to the uncertainty principle (and the hard no supra luminosity limit). It is not a point particle, but rather a ball of uncertainty. Within and around that region. This was the case at larger scale, even before determining that spacetime is discrete in a multi-fold universe. Spacetime reconstruction models show that spacetime, discrete as already mentioned, and particles are presented by microscopic black holes (possibly extremal for charged particles). The particle blackholes result from the structure of the effective potential surrounding every particle, as a result of the EPR entanglement of all the virtual particles that are emitted by the particle. Spacetime points are rather the result of the spacetime concretization by passage of particles following random walks. Massless carriers like photons are also Schwarzschild blackholes. All these are relevant only at very small scales, below the scales of typical quantum models. This model describes spacetime and gravitation as 2-D processes at very small scales and 4-D at larger scales. The spacetime is Lorentz invariant, thanks to the random walk-based spacetime reconstruction that generates a fractal structure and a non-commutative geometry. GR equation can be recovered as a result of the models of [1] (by computation of path integrals, or by Thermodynamics arguments).

The mechanisms of multi-folds, the main feature proposed in [1], trigger additional structures (folds) when particles are (EPR) entangled so that additional paths can traverse the folds, where the EPR entangled particles can always meet as a same exit points. Doing so, all the activated folds (i.e. multi-folds) create attractive potentials in $\frac{1}{r}$ in between the entangled particles $\left(\frac{1}{r^{2}}\right.$ per fold $)$ towards their source or center the mass, depending of the use cases and movements (and masses involved e.g. massive or massless). When involving virtual particles emitted by a source of energy, this potential is reminiscent of gravity. It can also be looked as adding contributions of the Ricci curvature scalar R of the folds, from all matter or energy contributions, to build a new Ricci curvature scalar field R and, with the direction of attraction information, a new consistent Ricci curvature tensor. Doing so, for all sources of energy, recovers Einstein's GR field equations (or Hilbert Einstein Action); which is amazing as invariance of surfaces (the real geometrical meaning behind the Hilbert Einstein Action) or variations of the Hilbert Einstein had, 
at no point, be postulated in [1] prior to that determination (something that can't exactly be said the same way for strings). Also, the multi-folds have a spin-2 symmetry.

This, as well as the microscopic black hole models, indicates that semi-classical models can be used till way smaller scales that usually expected (if tuned to behaviors described in [1]). Doing so, we can see particles positions, in the presence of entanglement and gravity, will oscillate preferably along the geodesics.

It reminds of few string features, but in appearance only:

- Superstring theory models particles as little strings instead of point particles. In a multi-fold universe of [1], the strings could rather be seen as the result of how particles appear as they wiggles back and forth predominantly along geodesics of choice, as a result of gravity (due to entanglement). It directly relates to the discussion in [1] about a similar observation made in [2] on the horizon of black holes (and the different variations of Black Hole soft hairs theorems). In [1], particles appear with their uncertainty regions that are shaped like wiggling strings in the presence of gravity.

- The length of the uncertainty walk on the geodesic is proportional to the energy of the particle. This is also the rule linking length and energy in string theory.

- At times, superstring theory models spacetime as the world sheets, building the manifold with these geodesics.

- When looked this way, the Nambu-Goto Action, behind all the string models, extremizes the world sheet surfaces (as soap bubbles minimize their surfaces between supports - here, the different string lines at different time - see [3]). Therefore, this action is immediately equivalent to the Hilbert Einstein Action, which also extremize surfaces. It is therefore no surprise that Nambu-Goto and strings recover exactly GR (when computing linear GR perturbations, i.e. conventional gravitons), at any order, or that, even with variations or extensions, they always recover or include some forms of graviton. Modeling gravitons and matching GR series expansions became the motivation, claim of fame and the main selling point for (super)strings as a theory of gravity and a Theory of Everything (ToE). Historically, some scattering amplitudes proposed ad hoc and computed for mesons gave good results. They were later understood and visualized, as strings linking quarks and anti-quarks in mesons (with the strong interactions increasing when the string is stretched; it was prior to QCD). The Nambu-Goto Action formalized the physics that includes minimizing surface of the world sheet to model the dynamics. More details and history are available in [4]. The point is that, in retrospect, the apparition of the graviton, not trivial to compute, was actually obvious. So yes, string models include gravitons (graviton-like particles) but it is really just because of how the world sheets are defined and then linked to spacetime. The same analysis can be repeated about (D-)branes.

- On the other hand, [1] does not start from a Hilbert Einstein Action (and variations or extensions) or from surface extremization Actions, or strings or analogous Physics. Yet by modeling EPR entanglement and a proposal to address the EPR paradox with multi-folds mechanisms, it ends up also with the GR field equation and the Hilbert Einstein Action (and spin-2 gravitons). One could argue that it is at least a similar feat as strings that should warrant some attention.

- It also means that spacetime in a multi-fold universe can contain string world sheets or can be modeled by superstrings or branes: spacetime shares equivalent Actions.

\subsection{Multi-fold universe and super string landscape and swampland}

In a multi-fold universe, entanglements activate multi-folds outside ${ }^{2}$ the spacetime [1]. Multi-folds attach to pairs of entangled particles (real or virtual) and paths in these folds become available to path integrals of particles

2 [1] could have decide to rather use wormholes (or folds) within spacetime (e.g. like ER bridges). All the mechanisms, models and features would remain valid, except that the AdS position (as tangent to spacetime and as in the AdS/CFT correspondence) may be different or more cumbersome to discuss and the analysis presented here may have to evolve a bit: superstring and multi-fold may lose, or have to adapt, some of their relationships detailed here. But, allowing this (in spacetime wormholes instead of outside spacetime) leads to two additional 
encountering a mapping to the folds. The result is the appearance in spacetime (in between the entangled particles) of an effective potential attractive towards the center of mass of mass and in $\frac{1}{r}$. It is equivalent to positive curvature (additive) contributions.

[1] Goes out of its way to emphasize that:

- $\quad$ Folds do not have to be governed by the Hilbert Einstein Action. They just might.

- Multi-folds live outside spacetime in a AdS(5) space.

- Multi-folds attached to entangled particles are the source of gravity like effects and spin-2 symmetries. When quantized (i.e. when discretized in [1]), the multi-folds match quantas of spacetime: they are the gravitons and they live in $\mathrm{AdS}(5)$; not our spacetime. It is their effects that appears through the effective potentials (or reconstructed curvature field) that make it look like they could live in our spacetime. The mapping is what transposes the effect to spacetime. This models is what ensure normalizability (no divergences) at the difference of conventional QFT approaches. It also shows that curvature can also be considered as a visualization tool more than a physical geometric reality.

- Gravity and gravitons can be massless or massive. Massive gravity correspond to entanglement of massive virtual particles pairs emitted by an energy source (i.e. particle). This effect only exist at very small scales, where it can become significant.

- Because of its bottom-up approach, multi-fold massive gravity does not suffer of the challenges and inconsistencies often met with a massive GR model [6]. Yet, we also know that such massive gravity can be made consistent [7]. However, note that [7] models massive gravity at large scale. This is not the result obtained in [1], which makes it matter only at very small scales, a fact easier to accept considering how GR has been repeatedly validated at very large scales.

- In general, additional entanglement also adds gravity like contributions.

- $\quad$ By construction, the background space can be modified from an initial condition by adding energy / matter to spacetime. These contributions are always with positive curvature. Spacetime is therefore either flat (without matter) or positively curved (unless if started with a negative initial condition for some reason). Negative curvature cannot be produced by the multi-fold mechanisms in [1] and so in [1], it is unphysical!

This matches interestingly many results in superstrings. To name a few:

- Gravitons are usually closed superstrings (bosonic loops of spin-2), that can live in AdS (+other dimensions) - and other spaces. It would explain why superstrings can model strong (strongly coupled) gravity.

- $\quad$ Superstrings models can exist in a large variety of ways (e.g. different configurations of the compact additional dimensions; usually characterized by different Calabi-Yau manifolds [23]). It led to the notions of string landscapes and swamplands where superstrings would be respectively viable and making physical sense, or not [19].

challenges: 1) folds probably would have to follow GR in our spacetime (maybe not but it is harder to argue). As explained in [1] that is a constraint that is limiting, yet not an impossibility. 2) ER bridges in our spacetime may be harder to comprehend when spanning macroscopic distances! Shouldn't they be observable? (2) is why while equivalent in terms of most of the results, [1] did not decide to pursue that path and just to assume it as a less interesting particular case. Yet a pre-print post publication of [1], shows that traversable wormholes of very small diameters could be envisaged in our spacetime [26]. Without any idea, if they would be detectable or observable or how things would encounter them and interact with them, it is hard to say it if would make sense as "in spacetime" (multi-)folds. But, if they are undetectable and [26] is correct, they could be candidates for a variations of multi-fold universe were multi-folds would be built with such wormholes and paths could traverse them. For now, we do not explore further or support this variation of multi-folds. It may be for future work. 
- The string landscape is so far empty of model matching our universe, even if expected, or prayed to be populated by some... At least no model has been found so far that can be thought to be suitably related to our universe.

- The string swampland is richly populated

- More than $10^{500}$ (or even $10^{272000}$, or more) types of theories are to be evaluated and classified!

- It has been shown that superstrings cannot be consistent or stable in a universe with positive dark energy and / or positive curvature like our universe.

Let us compare.

Many of these top level points match amazingly well [1], as multi-folds are sets of spheres (i.e. closed), in AdS(5) (i.e. negative curvature space); while generation of negative curvatures is not possible in spacetime but can be in a tangent space where multi-folds (and superstrings) and gravitons live. From the point of view of [1], it is perfectly logical and expected that strings only live in a space with negative curvature (e.g. AdS(5) ++) and influence spacetime through holography or AdS/CFT correspondence (see next section). In [1] that effects comes from the mappings.

In [1], with a model of particles (and spacetime locations) modeled as microscopic black holes (surrounding them), $\operatorname{AdS}(5)$ is tangent to our spacetime at every such points (let's not finesse on arguing about embedding space, tangent space or dual space). The microscopic black holes could also be seen as the sources and seeds of the multifolds [8]. But they also could be entertained as the place where the strings characterizing the particles are attached to spacetime, hinting at the superstring model. That picture also evolves a bit the way that spacetime and world sheets or branes should relate. World sheet may not be the spacetime, but their growth match spacetime creation or updates/perturbation as do the multi-folds. This point of view may possibly help better recover macroscopic spacetime in superstrings, today, still a challenge.

The onset of folds due to entanglement is also hinted in superstrings and in Physics in general [8.9]. It shows that the ideas of [1] may appear surprising, but they were already hinted by many conventional Physics models!

We will discuss the requirement for positive curvature for superstring in the next subsection.

\subsection{AdS / CFT Correspondence}

With AdS(5) tangent to spacetime, gravity is living in $\mathrm{AdS}(5)$ and impacting spacetime via attractive effective potentials (or curvatures) resulting from the mappings between spacetimes and folds. With gravity factually weaker in our spacetime than the other interactions (at normal quantum, semi classical and classical scales), [1] has recovered the AdS/CFT correspondence conjecture and the holographic principle.

In [1], it is no more a conjecture! It is a fact. CFT can be replaced by QFT, because [1] argues that with a discrete spacetime, background independence and torsion (both features of the model in[1]), gravity effects are well behaved (no singularity, no divergence, i.e. renormalizable: it is by default normalized). Again gravity is in AdS(5), but its effects are in our spacetime. [1] matches and go way beyond what superstrings tell us with the AdS/CFT correspondence.

Our spacetime is the boundary of AdS(5) and gravity lives in AdS(5) with effects, through the multi-folds mappings, in spacetime. However, remember that multi-folds do not necessarily have to obey the Hilbert Einstein Action for this to work. They might. We will repeat it again and again.

[1] and superstrings can share a same view of key aspects of the universe! 


\subsection{ER=EPR conjecture}

The ER = EPR conjecture showed another duality between connected black holes (à la ER bridge) and entangled blackholes. it is immediately reminiscent of the folds and mappings between EPR entangled particles in [1]. [1] was developed without even knowing about ER=EPR, that was discovered when compiling relevant prior works.

$\mathrm{ER}=\mathrm{EPR}$ usually models behaviors of black holes in AdS, where black holes have interesting properties or can be fully modeled. Yet traversability when the black holes are connected to form a wormhole is a problem: it often requires exotic matter, infinite time or the black holes or wormholes are unstable, especially when traversed. Multi-folds avoid these problems by not imposing Hilbert Einstein Action to define its dynamics.

[1] can allow paths of path integrals to traverse the folds. It is something that ER=EPR has not considered and therefore ER=EPR conjecture has missed, so far, the gravity generation (or modeling) impact of the approach. Our guess is that they did not pursue either because nobody made the connections of the plausible implications or because non-traversability when modeled by GR prevented paths from path integral to use the wormholes or travel through the connected black holes.

In fact, we also discovered, after publishing [1], that [10] proposed that, using the AdS/CFT correspondence and the holographic Schwinger effect at strong gravity coupling, and with superstrings living in AdS(5) (+ more dimensions), an entangled pairs of particles and anti-particles, quarks in their example, would automatically have a wormhole in $\mathrm{AdS}(5)$, along their world sheet, joining the two entangled particles (It is a superstring view of the world). It is the closest superstring and AdS/CFT endorsement of the multi-fold mechanisms that [1] proposes. That paper stopped short of all the consequences that result from the apparition of the wormhole. We also want to make sure that the reader remembers that, in [1] , we do not assume that the wormhole follows Hilbert Einstein type of action. Yet, this is a resounding illustration that what we propose was actually already contained or hinted in many models of conventional physics.

\subsection{Other similarities and differences}

\section{$\mathrm{GR}=\mathrm{QM}$}

The conjecture QR=QM [11], that "we'll observe quantum gravity using quantum computers in a lab sometime in the next decade or so". Somehow is seems very well aligned with [1] and one of its proposal for validation for multi-fold universe: [11] claims the above. [12] argues that quantum computing is universal and can be done by any type of entangled entities to obtain the same results. So indeed, gravity should appear not, as a simulation, but in between the entangled Qubits; because of the fundamental results obtained by [1] in terms of attraction between entangled systems. Of course, the question is when we will be able to detect that.

[1] concretizes, probably differently from what [11] really had in mind, how quantum computing will indeed allow us to observe quantum gravity!

As a note, [13] criticizes [11], but it may be a misunderstanding of what the author of GR=QM meant. From a Quantum computing and information point of view [12], our interpretation and outcome is what [11] meant! And it makes sense, at least in a context like [1].

\section{Black Holes and Singularities}

Superstrings have managed to address a whole bunch of singularity problems. Yet their models of black holes are essentially limited to AdS spaces. That may be useful to model wormholes and multi-folds for [1] if they were following GR derived equation (which, in [1], they might but don't have to). The exotic properties and closed form solutions of AdS black holes help address discussions like traversability, stability, exotic matter and time like loops. Yet they are not very useful in our spacetime, or in a space with a positive curvature space like dS (de Sitter), or with a positive cosmological constant, as it seems that our universe is. 
Interestingly, [1] also guarantee the absence of any gravity related singularity (i.e. in black holes and at the big bang or big crunch if it was to exists) because spacetime is discrete (so always at least on minimum length), because it can introduce torsion (at very small scales) within matter/energy that does not propagate but would prevent singularities [14] and probably also because of its dark energy and positive cosmological constant mechanisms ([1] may help explain these effects). As a result, it can support big bounces scenarios, if that was the cosmological evolution.

[1] also derives area laws for spacetime and for blackholes matching both blackhole theories and thermodynamics models for black holes, horizons and spacetime. For spacetime, these can be used to recover GR in a way well known since [15]. Several black hole paradoxes seem resolved, or at least well mitigated, in a multi-fold universe.

\section{Positively curved spacetime}

We live, apparently, in a flat or positively curved spacetime with dark energy (i.e. a positive cosmological constant) (Although this has been questioned, several times recently but with limited support [16]). On the other hand, it is now clear that superstrings cannot live in such a universe per [17]. These universes are part of the swampland.

The recently confirmed AdS instability for GR [18] is another indication that superstring can live in AdS but that our spacetime cannot be AdS. Otherwise, it would not be able to contain matter (macroscopically)! And yes, [1] states that matter are black holes but it is not exactly the intent of what [18] showed and why AdS is unstable under GR. In [1], this conclusions is not a result of instability: [1] does not and cannot apply to AdS, as it cannot physically generate negative curvature. An interesting consequence is the flip conclusion that matter does not exists (macroscopically) in AdS(5), the superstring universe. Therefore, besides gravitons, the other superstrings, appearing in superstring theories, do not describe particles in our space time, unless through D-branes world sheets or by connecting to our spacetime attached to particles that they characterizes. In [1] that would be connecting to spacetime through the microscopic black hole surrounding the particle and within its uncertainty region. And yes that uncertainty region may appear as a string per the above but is not, per this instability. More way to reconcile that with AdS/CFT correspondence are discussed in the speculative section 3.

[1] showed that it is ok that superstrings live in the tangent space with gravity impacting our spacetime via multifolds and mapping. It is our version of AdS/CFT correspondence and holographic principle. In our view, it is a more sensible result than all the still on-going attempts to transform superstrings results in AdS to guess the results in dS or other positive curved spacetime à la de Sitter. That has never really worked... All this should clarify landscapes and swampland for superstrings [19].

\section{Entanglement and Gravity}

QFT and Superstrings do not model well particles as discussed in [1]. They models fields. As a result they also do not model well entanglement between individual particles (and strings). Their models and methods are only statistical (Thermodynamics) through correlations and entanglement entropies. That is in part why these approaches cannot see gravity emerging the same way as in [1].

[1] suggests that superstring theory should evolve to support explicit modeling of entanglement between strings, e.g. as in [1]. So far, and using the AdS/CFT correspondence conjecture, ER=EPR and [10] are examples of a first possible steps. Approaches like [9] are other ones. Lessons and approaches with multi-folds as in [1] and this paper are also an input.

\section{Background independence}

[1] is covariant and background independent both in its top down analysis (first part of [1]) and it reconstruction (bottom-up).

QFT and superstrings are not background independent. Many have argued that it is a major issue that creates complexity, divergences and renormalization issues. [1] agrees with these arguments. 
The duality discussed here may inspire ways to address this problem in QFT and superstrings; albeit without a bottoms-up approach it is more challenging. Maybe superstring model should encompass a bottoms-up (re)constructive effort, where spacetime is built, not pre-supposed.

\section{Strange (Discrete and Noncommutative yet Lorentz invariant) Geometries}

Noncommutative geometries have been shown to appear in some superstring models. Noncommutative geometry often are associated to discrete spacetime, as in [1]. The non-commutativity is in fact a way to preserve Lorentz invariance; but its physical motivation can't be cleanly explained by superstrings (they are rather only as math results).

[1] shows how Lorentz invariance of spacetime is the result of fractals produced by random walks, at very small scales, as paths of the path integrals. Doing so, produces a Lorentz invariant spacetime, that is discrete, and where noncommutative geometry expresses suitably the Lorentz invariance through the commutators (that render the position operators fuzzy). Interestingly, [1] derives the noncommutativity of the position operators in AdS(5), because of the dynamics of the multi-folds. As the holographic (mappings) effects in spacetime matches these dynamics, spacetime must also be discrete. This is a fantastic result and it implies, per our duality, that superstring theory should consider that the universe is discrete with random then noncommutative geometry.

These properties of a discrete spacetime were well known already through different reasoning [20,21]. Yet, its implications for superstring, while sometimes compatible, do not seem to have been seriously pursued. The physical explanation obtained by [1] is also very unexpected and had not been matched in $[20,21]$ (i.e. they are not able to either why Lorentz invariant, it is rather respectively postulated or deducted from random distributions but then without explaining why random.) or other non-commutative, fractal or fractional spacetime theories! There are many theories and models proposing some of these features out there, typically without a detailed justification.

\section{Supersymmetries, supergravity and more}

[1] does not require or assume supersymmetries or supergravity. It would remain unaffected by their existence. Without requiring them, [1] avoids the problems of the absence of proton decays and absence of magnetic monopoles to name a few and yet still have options for an Ultimate Unification of interaction scenarios that survive the absence of these effects. [1] shows how gravity further precludes proton decay [5] and magnetic monopoles in multi-folds universes.

[1] treats $\operatorname{AdS(5)~as~an~external~space.~The~extra~dimensions~of~AdS(5)~are~not~visible~to~us~(only~to~paths~of~our~}$ constituting particles through curvature of the effect of attractive effective potential). It does not matter, if AdS(5) is complemented by additional compact dimensions to reach 10 or 11 dimensions, as needed respectively by superstrings and M-Theory. Being outside spacetime matters, as discussed in our footnote earlier: we can't explain macroscopic ER bridges in spacetime: they are not exactly observed. It also helps with the notions of nonobservability of entanglement [27]. It also explains non divergence and normalizability of the quantized gravity modeled in spacetime by [1].

\section{Supersymmetry only in $\operatorname{AdS}(5)(++)$ ?}

This section is speculative and should be treated as such. We thought along time if this section should be added. We do not want to distract from the analysis above that shows all the touch points between multi-folds universe introduced in [1] and superstrings. Because this section is more speculative and provocative on what might or might not be, it may distract and become the focus of all the attention. We decided to go ahead and not push these considerations to another paper, because it is really part of the analysis and its implications. We hope that the reader will approach the section in the same spirit. 
Maybe one should think about super partner particles differently, and it may be why they have never been observed: maybe super partners exist after all, but, like gravitons or multi-folds, they would only live in AdS(5), not in our spacetime. We already understand how gravitons relate both to our spacetime and AdS(5). As mentioned earlier, none other physical particle than the gravitons should live in the superstring space as AdS is unstable with matter. But nonphysical (e.g. virtual, or other concepts) might exist without problem and they are clearly encountered by superstring theories. This unconventional idea may then require new holographic principles if we expect super partners to have effects in our spacetime. Otherwise they only exist for the sanity of the superstrings but would be unphysical or unconnected to physical reality, which maybe that is what it is!

So, according to [1], multi-fold live outside our spacetime, in AdS(5). When quantized massive and massless gravitons live in AdS(5). This recovers a version of the AdS/CFT correspondence for multi-folds universes. If we exploit all the considerations on correspondence between superstrings and multi-fold mechanisms, it is natural to imagine that $\mathrm{AdS}(5)$ can of course by extended with multiple additional (compact) dimensions (which we will here conveniently denote as $\operatorname{AdS}\{5)(++)$; it is all outside our spacetime anyway. $\operatorname{AdS}(5)(++)$ is the space where superstrings or M-theory live (with one more dimension). The (multi-) folds are not affected and their dynamic do not have to follow GR in $\operatorname{AdS}(5)(++)$ : graviton can continue to behave the same even if they now are in $\operatorname{AdS}\{5)(++)$.

Yet the multi-fold mechanism, with its mapping, brings some paths of the particle crossing the support domains of the mapping, in the fold. In [1] the fold is treated as a extension of spacetime; so physics is as in the conventional world, only the spacetime is curved and the folds are single tenant (i.e. single instance per particle: no interactions in the folds other than at entry and exit. But if we relax a bit that last assumption, one could see that paths on the folds also brings the particle in $\mathrm{AdS}(5)(++)$, where Physics is now dictated by superstring theory (and it could explain why AdS/CFT may model aspects of our spacetime physics in the bulk, as announced earlier).

For the sake of discussion, let's see how that could happen and how could it play out. Two scenarios can maybe explain how some of these path would lead to strings:

1) because of uncertainties in our spacetime (and in the folds), the folds wiggle around tangent to spacetime. While the paths stays in their fold, the wiggling means that this implies wiggling around in $\operatorname{AdS}(5)(++)$, between the different positions of the fold spacetime.

2) at any point within the fold, the curvature effect results into an attractive effective potential felt in our spacetime. Then at the next time click, the fold evolves with the path. Yet if we consider an infinitesimal time with a given fold (at a given time), the path on the fold is a path left behind in $\operatorname{AdS}(5)(++)$.

In either cases (or combining both), these multi-folds of [1] leaves a myriad of tiny paths in $\operatorname{AdS}(5)(++)$. The particle (path) on it had all its physical properties with it. These could be seen (we have no better qualifier at this stage), as small strings in $\operatorname{AdS}(5)(++)$. These paths may be very small and proportional to the energy of the particle (in our spacetime) e.g. for option 2) or all of the same size, e.g. for 1)). In this model, it is clear that entanglement, and therefore gravity per [1], is responsible for the apparition of the tiny paths in $\mathrm{AdS}(5)(++)$ : no entanglement or no gravity and no mapping exists to make this happen. If just considering our spacetime, it also shows how superstring can affix themselves to particles while wiggling in $\operatorname{AdS}\{5)(++)$; if that was how particle in our spacetime get their properties, a claim solely from string theories.

When in $\operatorname{AdS}(5)(++)$, superstring Physics applies. Other particles exist (e.g. the super partners) depending on the superstring variant that is considered. They can interact with the tiny paths and do Physics with associated stringy Feynman diagrams. How that might in anyway be then reflected to our reality is anyone's guess and for future work, as symmetries in our spacetime and conservation rules must be suitably handled. At least this way, it may not matter that magnetic monopoles do not exist; they can just exist in $\operatorname{AdS}(5)(++)$. The handling of proton decay depends on how the above is handled or if proton stability could be a landscape requirement. Or physics in $\operatorname{AdS}(5)(++)$, while tacking place has no effect at all on our spacetime... In fact, even the requirement for symmetry 
breaking may be relaxed, as in $\operatorname{AdS}(5)(++)$, super partners could have the same mass as particles in our spacetime. Or physics in $\operatorname{AdS}(5)(++)$, while tacking place, has no effect at all on our spacetime...

Ensuring that symmetries in our spacetime and conservation rules must be suitably handled could be achieved by prescribing that

i) only interactions where super partners or others are virtual string account.

a. They can be modeled as reflected in our spacetime via the mappings (i.e. this way, the Feynman diagram has input and output with spacetime particles and the blob in between reflects the multi-folds and interactions in $\operatorname{AdS}(5)(++))$. As far as we know, none of these have been ever observed so far.

ii) supersymmetry accommodates not having proton decay, e.g. via gravity prevention of proton decay or supersymmetry conservation rules that amount to conservation of baryon and lepton numbers (not just their differences) or other mechanisms. This may include other prescriptions to protect other symmetries; but we do no

Of course, ii) is nontrivial but it would also bring conventional GUTs back in the race ${ }^{3}$. It is possible per [29,30,31]. This way, for superstrings: no proton decay, no magnetic monopoles (they are at best in $\operatorname{AdS}(5)(++))$, no recurrent mass acquisition in our spacetime, and no never observed super partners (they are also in $\mathrm{AdS}(5)(+))$. It is

These could be good criteria for the string landscape. The scheme above may not yet help for GUTs as the other effects including magnetic monopoles can't be address if we can't put the super partners outside our spacetime. What could be proposed for that is still unclear at this stage.

\section{Conclusions}

We believe that [1] makes a compelling case for the consistency of its multi-fold proposal. The present paper shows a duality between multi-fold universe and superstrings with an amazing amount of explanations and insights on what happens and what does not happen in superstrings, as well as what should be worth investigating to go to the next step and either converge superstrings with multi-fold universes or bring superstrings to the next level. It also helps programs like the AdS/CFT correspondence conjecture, the ER=EPR conjecture and GR=QM. As often, what are conjecture in superstrings are facts with a twist, or theorems in [1], or often offer some insight on what is meant or happening in superstrings with physical interpretation relating to spacetime. After all, unless spacetime and strings are suitably compartmentalized, as also proposed here, the fact that strings and matter live in different universes may hamper some superstring theories ambitions as ToEs (unless if our latest proposal for super partners resulted into something concrete and may be solves as a result also the issues of predicting proton decay or magnetic monopoles). AdS/CFT correspondence, as a conjecture, today may miss the oomph that [1] provides to close the deal.

Maybe [1] can also help with aspects of the landscape or even the elusive M-theory.

[1] also offers touch points with the superstrings evil twins (depending on those perspective - It is a figure of speech; we do not make judgement of which is what): Loop Quantum Gravity (LQG) and other spacetime construction approaches (e.g. [22]). For example, the reconstruction schemes and entanglement models and mechanisms of [1] are key input.

[1] shows also significant impact on the Standard Model, when we add gravity (especially the short scale massive contributions). It could contribute explanations to several famous open issues.

\footnotetext{
${ }^{3}$ With for examples supersymmetry only for virtual particles (and no proton decay). Feasibility and implications have not been evaluated. It is just an idea.
} 
Despite all the energy out there, it is fair to say that the momentum behind quantum gravity, GUTs and ToEs, and that includes superstrings, has again waned. At least, it appears so from the outside.

We certainly would invite some opportunities to put it all together, as this paper started to do by positioning what [1] and its multi-folds mechanisms can offer to superstring, M-theory as quantum gravity in general. In fact [1], with its massive gravity contributions at small scale seems to offer a new alternative for a Unification of all the interactions by democratizing the effects of all interactions, at very smalls scales, instead of uber symmetry approaches à la Electroweak that first combined it with the strong interaction into GUTs [28]; something that has run into some significant snags.

Although quite different approaches, all the models depicts facets of the required framework. Hopefully, this paper and [1] helped, and maybe these approaches can progress more collaboratively together?

References: (most references come from popular science to make the discussion more approachable) [1]: Stephane H. Maes, (2020) "Quantum Gravity Emergence from Entanglement in a Multi-Fold Universe", viXra:2006.0088v1, (June 9, 2020).

[2]: G. 't Hooft, (1990), "The Black Hole Interpretation of String Theory", Nuclear Physics B335 (1990) 138-154

[3]: Zwiebach, Barton (2003). "A First Course in String Theory". Cambridge University Press.

[4]: https://en.wikipedia.org/wiki/History_of string theory

[5]: Stephane H Maes, (2020), "Gravity Induced Anomalies Smearing in Standard Model so that Protons May Never Decay, Except in Black Holes ", viXra:2006.0128v1, https://shmaesphysics.wordpress.com/2020/06/12/protonsmay-never-decay-except-in-black-holes/, June 12, 2020.

[6]: Claudia de Rham, (2014), "Massive Gravity", arXiv:1401.4173v2

[7]: Claudia de Rham, Gregory Gabadadze, Andrew J. Tolley, (2010), "Resummation of Massive Gravity", arXiv:1011.1232v2

[8]: ChunJun Cao, Sean M. Carroll, Spyridon Michalakis, (2016). "Space from Hilbert Space: Recovering Geometry from Bulk Entanglement", arXiv:1606.08444v3.

[9]: van Raamsdonk, Mark (2010). "Building up spacetime with quantum entanglement", Gen. Rel. Grav. 42 (14):

2323-2329. arXiv:1005.3035

[10]: Julian Sonner, (2013), "Holographic Schwinger Effect and the Geometry of Entanglement", arXiv:1307.6850v3

[11]: Leonard Susskind, (2017). "Dear Qubitzers, GR=QM", arXiv:1708.03040

[12]: Seth Lloyd, (2006), "Programming the Universe", Alfred A. Knopf

[13]: https://motls.blogspot.com/2017/08/gram-paper-shows-susskind-isnt-real-co.html

[14]: A. Trautman, (1973), "Spin and Torsion May avert Gravitational Singularities", Nature Physical Science, ol.

142, 7-8.

[15]: Ted Jacobson, (1995), "Thermodynamics of Spacetime: The Einstein Equation of State", arXiv:grqc/9504004v2.

[16]: https://www.mpls.ox.ac.uk/news/new-research-casts-doubt-on-the-accelerating-expansion-of-the-universe

[17]: Georges Obied, Hirosi Ooguri, Lev Spodyneiko, Cumrun Vafa, (2018), "De Sitter Space and the Swampland", arXiv:1806.08362v3.

[18]: Georgios Moschidis, (2018), "A proof of the instability of AdS for the Einstein--massless Vlasov system", arXiv:1812.04268v1.

[19]: Cumrun Vafa, (2005), "The String Landscape and the Swampland", arXiv:hep-th/0509212v2

[20]: S. Doplicher, K. Fredenhagen and J. E. Roberts, (1994), "Spacetime quantization induced by classical gravity", Phys. Rev. B 331 (1994) 33.

[21]: Hooft, Gerard 't, (2016), "How quantization of gravity leads to a discrete space-time", J. Phys.: Conf. Ser. 701 012014

[22]: Johannes Thueringen, (2015), "Discrete quantum geometries and their effective dimension", Ph.D. Thesis, Humboldt-Universitaet zu Berlin

[23]: https://en.wikipedia.org/wiki/Calabi\%E2\%80\%93Yau manifold 
[24]: https://en.wikipedia.org/wiki/Reissner\%E2\%80\%93Nordstr\%C3\%B6m metric

[25]: https://en.wikipedia.org/wiki/Kerr-Newman metric

[26]: R. Moti, A. Shojai, "Traversability of quantum improved wormhole solution", arXiv:2006.06190v1

[27]: Ning Bao and Jason Pollack and Grant N. Remmen, (2015), "Wormhole and entanglement (non-)detection in the ER=EPR correspondence", arXiv:1509.05426.

[28]: Stephane H Maes, (2020), "Ultimate Unification: Gravity-led Democracy vs. Uber-Symmetries", viXra:2006.0211v1, https://shmaesphysics.wordpress.com/2020/06/16/ultimate-unification-gravity-leddemocracy-vs-uber-symmetries/, June 16, 2020.

[29]: Jogesh C. Pati, (1996), "Baryon Non-Conservation in Unified Theories, in the Light of Supersymmetry and Superstrings", arXiv:hep-ph/9611371v1

[30]: https://ncatlab.org/nlab/show/proton+decay

[31]: G. Lazarides, C. Panagiotakopoulos, Q. Shafi, (1993), "Supersymmetric Unification without Proton Decay", arXiv:hep-ph/9306332v1

[32]: Burinskii, Alexander, (2008), "The Dirac-Kerr-Newman electron", arXiv:0507109v4 